\title{
THE COSMIC HAUSDORFF TOPOLOGY, THE BOUNDED HAUSDORFF TOPOLOGY AND CONTINUITY OF POLARITY
}

\author{
JEAN-PAUL PENOT
}

\author{
(Communicated by William J. Davis)
}

\begin{abstract}
We give geometric proofs of recent results of G. Beer [13]: the Young-Fenchel correspondence $f \rightarrow f^{*}$ is bicontinuous on the space of closed proper convex functions on a normed vector space $X$ endowed with the epidistance topology and the polarity operation is continuous on the space of closed convex subsets of $X$ with the bounded Hausdorff topology. Our methods are in the spirit of a famous result due to Walkup and Wets [31] about the isometric character of the polarity for closed convex cones. We also prove that the Hausdorff distance associated with the cosmic distance on the space of convex subsets of a normed vector space induces the bounded -Hausdorff topology. This shows a link between Beer's results and the continuity results of [2].
\end{abstract}

There are two methods to define topologies on the space $\mathscr{C}(X)$ of nonempty convex subsets of a normed vector space $X$ and on the set $\Gamma_{0}(X)$ of closed proper convex functions on $X$. In the first, functions are the primitive objects, and facts about convex sets are deduced as corollaries of results for convex functions, identifying a convex set with its indicator function $[1-3,10-14,21$, $24, \ldots$ ]. In the second approach, the basic objects are convex sets, especially convex cones, and convex functions are identified with their epigraphs $[2,4$, $5,14,21,24, \ldots]$. The first approach is in order when one defines a topology through a regularization process as in $[1,2, \ldots]$. The second one is more natural when the topology is first defined on $\mathscr{C}(X)$.

This is the case in a series of recent works starting with [27]: [4-9, 28] which has given a new interest to a topology introduced in $[24,25]$ on the space $\mathscr{C}(X)$ of nonempty convex subsets of a Banach space $X$ called here the bounded Hausdorff topology or the epi-distance topology. Here we give a direct proof that the polarity mapping is a continuous correspondence for this topology, a result established by G. Beer [13, Theorem 3.6] by analytic methods. We deduce this property from a pioneering result due to D. W. Walkup and

Received by the editors January 4, 1990 and, in revised form, July 15, 1990.

1980 Mathematics Subject Classification (1985 Revision). Primary 54B20; Secondary 52A05, $54 \mathrm{~A} 20$.

Key words and phrases. Bounded Hausdorff topology, bounded hemi-convergence, convex sets, conjugacy, cosmic distance, epi-distance, Legendre-Fenchel transform, polarity. 
R. J.-B. Wets [31] dealing with a similar property for convex cones. Thus our task is reduced to showing that taking the conical hull of a set and taking the intersection of a convex cone with a hyperplane not containing the origin are continuous operations. These results may be useful elsewhere; the corresponding facts using the Mosco convergence instead of the bounded Hausdorff topology have been studied in detail in $[16,17,21,22,25]$. Identifying a function with its epigraph and using a geometrical argument as in [17] we recover the result of G. Beer [13, Theorem 3.5] about the continuity of the Fenchel correspondence with respect to the epi-distance topology.

We prove a similar result for a topology introduced by $R$. T. Rockafellar and R. J.-B. Wets, the cosmic topology. This fact was shown to be valid in Hilbert spaces by H. Attouch and R. J.-B. Wets [2]. More importantly, we show that the cosmic topology coincides with the bounded Hausdorff topology. Since it is proved elsewhere [28] that the latter topology coincides with the topology obtained by regularization, this reinforces the interest of the cosmic topology and of its relatives and shows in particular that the usual operations are continuous with respect to the cosmic topology, provided qualification conditions are satisfied (see $[7,8,27])$.

\section{CONTINUITY OF THE CONICAL HULl OPERATOR}

Given a normed vector space (n.v.s.) $X$ with dual space $X^{*}$ we denote by $B$ (resp. $B^{*}$ ) the closed unit ball of $X$ (resp. $\left.X^{*}\right)$. The Hausdorff excess of a subset $C$ of $X$ over a subset $D$ of $X$ is given by

$$
\begin{aligned}
e(C, D) & =\inf \left\{r \in \mathbb{R}_{+}: C \subset D+r B\right\} \\
& =\sup \{d(x, D): x \in C\},
\end{aligned}
$$

where $d(x, D)=\inf \{d(x, y): y \in D\}$ (with the usual convention $\inf \varnothing=$ $+\infty, \sup \varnothing=0$ in $\mathbb{R}_{+}$) (see [15]) and the (Pompeiu-) Hausdorff distance of $C$ and $D$ is given by

$$
d(C, D)=\max (e(C, D), e(D, C)) .
$$

For $r \in \mathbb{R}_{+}$we set

$$
\begin{aligned}
& e_{r}(C, D)=e(C \cap r B, D), \\
& d_{r}(C, D)=\max \left(e_{r}(C, D), e_{r}(D, C)\right) .
\end{aligned}
$$

The bounded Hausdorff topology on the set $\mathscr{C}(X)$ of nonempty convex subsets of $X$ is the topology generated by the family

$$
B_{r}(C, \varepsilon)=\left\{D \in \mathscr{C}(X): d_{r}(C, D)<\varepsilon\right\} \quad(r, \varepsilon) \in \mathbb{P}^{2},
$$

where $\mathbb{P}$ is the set of positive numbers. Setting for $C, D \subset X$,

$$
\delta_{r}(C, D)=\sup _{r B}|d(\cdot, C)-d(\cdot, D)|
$$

one has $[4,6-8,28]$ for $r>r_{0}=\max (d(0, C), d(0, D))$,

$$
d_{r}(C, D) \leq \delta_{r}(C, D) \leq d_{3 r}(C, D)
$$


so that one gets that the restriction of this topology to the set $\mathscr{C}_{0}(X)$ of nonempty closed convex subsets of $X$ is metrizable with associated metric given by

$$
\delta(C, D)=\sum_{n=1}^{\infty} 2^{-n} \delta_{n}(C, D)\left(1+\delta_{n}(C, D)\right)^{-1} .
$$

Considering the set $\Gamma_{0}(X)$ of closed proper convex functions on $X$ as a subset of $\mathscr{C}_{0}(X \times \mathbb{R})$ by identifying a function with its epigraph, we get a topology on $\Gamma_{0}(X)$ known as the bounded Hausdorff topology [27, 28] or the epi-distance topology [4] or the topology of bounded hemi-convergence. The following result is quite easy. In finite dimensions it coincides with [17, Proposition 3.2].

1.1. Proposition. Let $H$ be a closed hyperplane of $X$ not containing zero. Then the mapping $Q \rightarrow Q \cap H$ is continuous from the set $\mathscr{Q}_{H}(X)$ of convex cones of $X$ whose intersections with $H$ are nonempty into $\mathscr{C}(X)$.

Since the bounded Hausdorff topology is unchanged when one replaces the norm by an equivalent norm, the preceding proposition is a direct consequence of the following more precise result (identifying $X$ with $X_{0} \times \mathbb{R}$, where $X_{0}$ is the hyperplane parallel to $H$ and containing 0 ). Here we endow a product with the supremum norm.

1.2. Proposition. Let $P, Q$ be convex cones of $X \times R$ whose intersections with $X_{1}=X \times\{1\}$ are nonempty and let $C, D$ be the convex sets given by $C \times\{1\}=$ $P \cap X_{1}, D \times\{1\}=Q \cap X_{1}$. Then, if $r \geq 1$ and $d_{r}(P, Q)<1 / 2$, one has

$$
d_{r}(C, D) \leq 2(1+r) d_{r}(P, Q) \text {. }
$$

Proof. Let us prove more generally that for any $c>1$ one has

$$
d_{r}(C, D) \leq c(1+r) d_{r}(P, Q)
$$

provided $d_{r}(P, Q)<1-c^{-1}$, that will give the result for $c=2$. Let $\delta>$ $d_{r}(P, Q)$ with $\delta<1-c^{-1}$. Since $r \geq 1$, for any $x \in C \cap r B$ we have $d((x, 1), Q)<\delta$ so that we can find $(y, t) \in Q$ with $\|y-x\|<\delta,|t-1|<\delta$. Then $t>1-\delta>c^{-1}$ and

$$
\begin{aligned}
\left\|t^{-1} y-x\right\| & \leq t^{-1}\|y-x\|+\left|t^{-1}-1\right|\|x\| \\
& \leq c \delta(1+r) .
\end{aligned}
$$

As $\delta$ is arbitrarily close to $d_{r}(P, Q)$ we get the result.

The continuity of the conical hull operator is less immediate.

1.3. Proposition. The mapping $R: C \rightarrow R(C):=\mathbb{R}_{+}(C \times\{1\})$ is continuous from $\mathscr{C}(X)$ into $\mathscr{C}(X \times \mathbb{R})$.

Proof. (a) Let $C \in \mathscr{C}(X)$ and let $\varepsilon>0, r \in \mathbb{R}_{+}$be given. Let us find $\delta>0$, $q \in \mathbb{R}_{+}$such that for $e_{q}(C, D)<\delta$ we have $e_{r}(R(C), R(D)) \leq \varepsilon$. Let us choose $r_{0}>d(0, C), x_{0} \in C$ with $\left\|x_{0}\right\|<r_{0}$. We may suppose $r \geq r_{0} \geq 1, \varepsilon \leq 1$.

Let us take $q=3 \varepsilon^{-1} r r_{0}+r_{0}, \delta=r^{-1} \varepsilon$. Since $R(C)$ and $R(D)$ are cones it suffices to show that for any $(x, s) \in R(C)$ with $\|(x, s)\|=r$ we have 
$d((x, s), R(D)) \leq \varepsilon$. Let us first suppose $s \geq r q^{-1}$. Then $s^{-1} x \in C \cap q B$ so that we can find $y \in D$ with $\left\|s^{-1} x-y\right\|<\delta$. Thus $(s y, s) \in R(D)$ and $d((x, s),(s y, s))<s \delta \leq r \delta \leq \varepsilon$.

Now let us suppose $s<r q^{-1}$. As $q \geq 1, \max (\|x\|,|s|)=r$, we have $\|x\|=r$. Let $t:=s\left(q-r_{0}\right)\left(r+s r_{0}\right)^{-1}, w:=(1-t) x_{0}+t s^{-1} x \in C$ since $C$ is convex and $t \in[0,1]$. Moreover

$$
\|w\| \leq\left\|x_{0}\right\|+t\left\|s^{-1} x-x_{0}\right\| \leq r_{0}+t\left(s^{-1} r+r_{0}\right)=q
$$

so that we can find $z \in D$ with $\|w-z\|<\delta$. Then $r\left(q-r_{0}\right)^{-1}(z, 1) \in R(D)$ and

$$
\begin{aligned}
&\left|r\left(q-r_{0}\right)^{-1}-s\right| \leq r\left(q-r_{0}\right)^{-1}+s \leq \varepsilon / 3+r q^{-1} \leq \varepsilon \\
&\left\|r\left(q-r_{0}\right)^{-1} z-x\right\| \leq r\left(q-r_{0}\right)^{-1}\|z-w\|+r\left(q-r_{0}\right)^{-1}(1-t)\left\|x_{0}\right\| \\
&+\left|r\left(q-r_{0}\right)^{-1} t s^{-1}-1\right|\|x\| \\
&<(1 / 3) \varepsilon r_{0}^{-1}\left(\delta+r_{0}\right)+r_{0} s\left(r+r_{0} s\right)^{-1} r \\
& \leq(2 / 3) \varepsilon+r_{0} s r \leq(2 / 3) \varepsilon+r_{0} r q^{-1} \leq \varepsilon .
\end{aligned}
$$

(b) Now let us suppose $d_{q}(C, D)<\delta^{\prime}$ where $\delta^{\prime}=\min \left(\delta, r_{0}-\left\|x_{0}\right\|\right)$. As $\left\|x_{0}\right\|<r_{0}<q$ we can find $y_{0} \in D$ with $\left\|x_{0}-y_{0}\right\|<\delta^{\prime}$, hence $y_{0} \in D \cap r_{0} B$. Thus we can interchange $C$ and $D$ in what precedes, taking $y_{0}$ instead of $x_{0}$, so that $e_{r}(R(C), R(D))<\varepsilon$, hence $d_{r}(R(C), R(D))<\varepsilon$.

Corollary. Let $H$ be a closed hyperplane of $X$ not containing 0 and let $\mathscr{B}_{H}(X)$ be the set of convex cones of $X$ with a base contained in $H$. Then the mapping $Q \rightarrow Q \cap H$ is a homeomorphism of $\mathscr{B}_{H}(X)$ onto $\mathscr{C}(H)$, where $\mathscr{C}(H)$ is the set of $C \in \mathscr{C}(X)$ with $C \subset H$.

Let us denote by $\operatorname{cl} A$ the closure of a set $A$.

1.5. Corollary. The mapping $C \rightarrow \operatorname{cl} R(C)$, where $R(C)=\mathbb{R}_{+}(C \times\{1\})$, is a homeomorphism of $\mathscr{C}_{0}(X)$ onto the set $\mathscr{Q}_{0}(X \times \mathbb{R})$ of closed convex cones of $X \times \mathbb{R}_{+}$whose intersections with $X \times\{1\}$ are nonempty.

Proof. Since for any cones $P, Q$ of $X \times \mathbb{R}$ one has $d_{r}(P, Q)=d_{r}(\operatorname{cl} P, \operatorname{cl} Q)$, it suffices to prove that $C \rightarrow \operatorname{cl} R(C)$ is a bijection. For any $C \in \mathscr{C}_{0}(X)$ one has $C \times\{1\}=(\operatorname{cl} R(C)) \cap X \times\{1\}$ since when $(x, 1)=\lim t_{n}\left(x_{n}, 1\right)$ with $t_{n}$ in $\mathbb{R}_{+}, x_{n}$ in $C$, we have $\lim t_{n}=1, x=\lim x_{n} \in C$. Moreover for each $Q \in \mathscr{Q}_{0}(X \times \mathbb{R})$ we have

$$
Q=\operatorname{cl}\left(\mathbb{R}_{+}(C \times\{1\})\right),
$$

where $C \times\{1\}=Q \cap X \times\{1\}$. This follows from the fact that for any $z \in Q$ one has $z=\lim \left(z+n^{-1} z_{0}\right)$ with $z_{0} \in C \times\{1\}$ arbitrary and $z+n^{-1} z_{0} \in R(C)$ for each $n \in \mathbb{N} \backslash\{0\}$. 


\section{CONTINUITY OF POLARITY}

Our starting point is the isometric character of the polarity for closed convex cones established by D. W. Walkup and R. J.-B. Wets [31] in the reflexive case. We give a new proof due to M. Volle [30] of this result without the reflexivity condition; it includes an isometric property for the excess $e_{r}$. It relies on the following lemma which extends [20] and [15, Theorem II.18] to the case of unbounded sets and [2, Lemma 3.13] to the nonreflexive case. Here we suppose $X$ and $Y$ are normed vector spaces (n.v.s.) in metric duality [18]; this means that the coupling functional $\langle\cdot, \cdot\rangle: X \times Y \rightarrow \mathbb{R}$ is such that, denoting by $B$ (resp. $B^{\prime}$ ) the unit ball of $X$ (resp. $Y$ )

$$
\begin{array}{ll}
\|x\|=\sup \left\{\langle x, y\rangle: y \in B^{\prime}\right\} & \forall x \in X, \\
\|y\|=\sup \{\langle x, y\rangle: x \in B\} & \forall y \in Y .
\end{array}
$$

Thus $Y$ can be identified with a subspace of $X^{*}$. For $f: X \rightarrow \mathbb{R} \cup\{-\infty,+\infty\}$ we set $f^{*}(y)=\sup \{\langle x, y\rangle-f(x): x \in X\}$. Let us recall that the support function of a subset $C$ of $X$ is the function $h_{C}:=h(C, \cdot)=\left(\psi_{C}\right)^{*}$, where $\psi_{C}$ is the indicator function of $C$ given by $\psi_{C}\left|C=0, \psi_{C}\right| X \backslash C=+\infty$; thus $h_{C}(y)=\sup \{\langle x, y\rangle: x \in C\}$. We use the extension to $\overline{\mathbb{R}}=\mathbb{R} \cup\{-\infty,+\infty\}$ of the addition of $\mathbb{R}$ obtained by setting $r \dot{+} s=+\infty$ as soon as $r$ or $s=+\infty$, $r \dot{+} s=-\infty$ for $r=-\infty$ and $s \neq+\infty$; we set $r+s=-((-r) \dot{+}(-s)), r-s=$ $-((-r) \dot{+} s)$. The infimal convolution (or epi-sum) of the extended real-valued functions $f, g$ on $X$ is given by

$$
(f \square g)(x)=\inf \{f(x-w)+g(w): w \in X\} .
$$

2.1. Lemma. For any nonempty subsets $C, D$ of $X$ with $D$ convex one has

$$
e(C, D)=\sup \left\{h_{C}(y)-h_{D}(y): y \in B^{\prime}\right\}
$$

Proof. This follows from the Toland's formula (see [19, (2.8) for instance]): for $f: X \rightarrow \overline{\mathbb{R}}, g: X \rightarrow \overline{\mathbb{R}}$, with $g$ closed proper convex,

$$
\sup (f-g)=\sup \left(g^{*} \div f^{*}\right),
$$

taking $f=d(\cdot, D), \quad g=\psi_{C}$ and observing that $f^{*}=\left(\|\| \square \psi_{D}\right) A^{*}=\psi_{B}+$ $h_{D}$.

Let us suppose in the sequel that $X$ is an arbitrary n.v.s. and that the mapping $y \rightarrow\langle\cdot, y\rangle$ defines a bijection of $Y$ onto the dual space $X^{*}$ of $X$. For $C \subset X$ we set $C^{\circ}=\{y \in Y: \forall x \in C\langle x, y\rangle \leq 1\}$. Let us observe that when $C$ is a cone we can replace 1 by 0 in this definition.

2.2. Proposition [30, 31]. Given two nonempty convex cones $P, Q$ of $X$ one has for each $r \in \mathbb{R}_{+}$

$$
\begin{aligned}
& e_{r}(P, Q)=e_{r}\left(Q^{\circ}, P^{\circ}\right) \\
& d_{r}(P, Q)=d_{r}\left(P^{\circ}, Q^{\circ}\right)
\end{aligned}
$$


Proof. It suffices to prove the first assertion and to suppose $r=1$, since $e_{r}(P, Q)=r e_{1}(P, Q)$. Since $h_{Q}=\psi_{Q^{\circ}}$, Lemma 2.1 shows that

$$
\begin{aligned}
e(P \cap B, Q) & =\sup \left\{h_{P \cap B}(y)-h_{Q}(y): y \in B\right\} \\
& =\sup \left\{h_{P \cap B}(y): y \in Q^{\circ} \cap B\right\} .
\end{aligned}
$$

Now it is well known [23, p. 136 for instance] that

$$
d\left(\cdot, P^{\circ}\right)=h_{P \cap B}
$$

in fact, as $\psi_{B}$ is continuous at 0 this follows from the equalities

$$
h_{P \cap B}=\left(\psi_{P}+\psi_{B}\right)^{*}=\psi_{P^{\circ}} \square\|\|=d\left(\cdot, P^{\circ}\right) .
$$

Therefore

$$
e(P \cap B, Q)=e\left(Q^{\circ} \cap B, P^{\circ}\right) .
$$

We are ready for our main result.

2.3. Theorem. For any n.v.s. $X$ the polarity $C \rightarrow C^{\circ}$ is a continuous mapping from $\mathscr{C}(X)$ into $\mathscr{C}\left(X^{*}\right)$.

Proof. Putting $X \times R$ in metric duality with $X^{*} \times \mathbb{R}$ by

$$
\langle(x, s),(y, t)\rangle=\langle x, y\rangle-s t
$$

we have

$$
C^{\circ} \times\{1\}=(R(C))^{\circ} \cap X^{*} \times\{1\},
$$

where $R(C)=\mathbb{R}_{+}(C \times\{1\})$. The result follows from Propositions 1.2, 1.3, and 2.2 .

2.4. Corollary. For any n.v.s. $X$ the Fenchel correspondence $f \rightarrow f^{*}$ is continuous from $\Gamma_{0}(X)$ into $\Gamma_{0}\left(X^{*}\right)$.

Proof. Putting $X \times \mathbb{R} \times \mathbb{R}$ in metric duality with $X^{*} \times \mathbb{R} \times \mathbb{R}$ by

$$
\langle(x, p, q),(y, s, t)\rangle=\langle x, y\rangle-p t-q s
$$

we observe that the epigraph epi $f^{*}$ of $f^{*}$ is given by the formula [17, Lemma $4.3 ; 29$, Theorem $14.4 ; 32$ ]

$$
\text { epi } f^{*} \times\{1\}=(R(\text { epi } f))^{\circ} \cap X^{*} \times \mathbb{R} \times\{1\},
$$

and the result follows from Propositions 1.2, 1.3, and 2.2 as above.

\section{IDENTIFYING THE COSMIC HAUSDORFF TOPOLOGY}

In a book in preparation Rockafellar and Wets introduce the notion of cosmic distance on a finite-dimensional Euclidean space or rather on the projective space associated with it. It is extended in [2] to the case of an arbitrary n.v.s. Let us recall their construction, omitting certain subtleties, as we do not need to define distances between directions and as we do not wish to give a precise definition of the geodesic distance on the sphere. 
3.1. Definition. Given a n.v.s. $(X,\|\|)$ the cosmic distance $d^{c}(x, y)$ between two points $x, y$ of $X$ is the Hausdorff distance between the intersections with the unit ball $B_{X \times \mathbb{R}}$ of $X \times \mathbb{R}$ of the rays $R(x)=\mathbb{R}_{+}(\{x\} \times\{1\}), R(y)=$ $\mathbb{R}_{+}(\{y\} \times\{1\})$ generated by $(x, 1)$ and $(y, 1)$ in $X \times \mathbb{R}:$

$$
\begin{aligned}
d^{c}(x, y) & =d\left(R(x) \cap B_{X \times \mathbb{R}}, R(y) \cap B_{X \times \mathbb{R}}\right) \\
& =d([0,1] \hat{x},[0,1] \hat{y}),
\end{aligned}
$$

where $\hat{x}=\|(x, 1)\|^{-1}(x, 1)$ and $\hat{y}$ has a similar meaning. Here and in the sequel we endow $X \times \mathbb{R}$ with the norm given by

$$
\|(x, r)\|=\left(\|x\|^{2}+r^{2}\right)^{1 / 2} \text {. }
$$

We need a variant of relation (1.1) for convex sets $C, D$ which involves $d_{r}(C, D)$ and not $d_{3 r}(C, D)$; it appeared in [4, Proposition 1.4]. The proof we present here for the sake of completeness avoids the use of duality and its first part (the one we need) is particularly simple. In the sequel, for a subset $C$ of $X$ we set $C_{r}=C \cap r B_{X}$.

\subsection{Lemma.}

(a) If $C, D$ are convex subsets of $X$ containing 0 one has for any $r>0$

$$
d_{r}(C, D) \leq d\left(C_{r}, D_{r}\right) \leq 2 d_{r}(C, D) .
$$

(b) For any convex subset $D$ of $X$ and any $s \geq 0, r \geq q>d(0, D)$ one has

$$
e\left(D_{r+s}, D_{r}\right) \leq(r-q)^{-1}(r+q) s .
$$

(c) For any convex subsets $C, D$ of $X$ and any $r>r_{0}:=\max (d(0, C)$, $d(0, D))$ one has

$$
d_{r}(C, D) \leq d\left(C_{r}, D_{r}\right) \leq 2 r\left(r-r_{0}\right) d_{r}(C, D) .
$$

Proof. (a) The first inequality is obvious as $e_{r}(C, D) \leq e\left(C_{r}, D_{r}\right)$ since $D_{r} \subset$ $D$. Now, given $s>d_{r}(C, D)$ and $x \in C_{r}$ we can find $y \in D$ with $d(x, y)<s$, hence $y \in D_{r+s}$. Then $z:=r(r+s)^{-1} y \in D_{r}$ and $d(x, z) \leq d(x, y)+d(y, z) \leq$ $2 s$, so that $e\left(C_{r}, D_{r}\right) \leq 2 s$. Interchanging $C_{r}$ and $D_{r}$ and taking the infimum on $s>d_{r}(C, D)$ the result follows.

(b) This can be seen as a particular case of [26, Theorem 5.1] by considering the multifunction $F$ from $\mathbb{R}$ into $X$ given by $F(r)=D \cap r B_{X}$ for $r$ in $\mathbb{R}_{+}, F(r)=\varnothing$ for $r$ in $\mathbb{R} \backslash \mathbb{R}_{+}$, but we prefer to give a direct proof. Let $x \in$ $D_{r+s}$. As $q>d(0, D)$ we can find some $x_{0}$ in $D_{q}$. Let $t=s(r+s-q)^{-1} \in$ $[0,1]$, so that $r=t q+(1-t)(r+s)$. Then $x^{\prime}:=t x_{0}+(1-t) x \in D_{r}$ and $\left\|x^{\prime}-x\right\|=t\left\|x-x_{0}\right\| \leq s(r+s-q)^{-1}(r+s+q) \leq s(r-q)^{-1}(r+q)$, as $u \rightarrow(r+u-q)^{-1}(r+u+q)$ is decreasing on $\mathbb{R}_{+}$. Assertion (b) follows. 
(c) Again the first inequality is obvious. The second one follows from (b) and the following inequalities in which $q>r_{0}, s>d_{r}(C, D)$ :

$$
\begin{aligned}
e\left(C_{r}, D_{r}\right) & \leq e\left(C_{r}, D_{r+s}\right)+e\left(D_{r+s}, D_{r}\right) \\
& \leq s+(r-q)^{-1}(r+q) s=2 r(r-q)^{-1} s .
\end{aligned}
$$

3.3. Remark. The inequality $d\left(C_{r}, D_{r}\right) \leq a d_{r}(C, D)$ with $a<2$ is not always true. Take $X=\mathbb{R}^{2}$ with

$$
\|z\|=|x|+|y| \text { for } z=(x, y),
$$

$C=\mathbb{R}_{+} \times\{0\}, D=\left\{\left(x,\left(1-r_{0}\right) x+r_{0}\right): x \in \mathbb{R}_{+}\right\}$with $r_{0}>0, r_{0}<1$ such that $2\left(2-r_{0}\right)^{-1}>a$. Then for $r=1$ one has $d\left(C_{r}, D_{r}\right)=2\left(2-r_{0}\right)^{-1}>a$ and $d_{r}(C, D)=1$.

Let us now connect the Hausdorff distance associated with the cosmic distance to the usual Hausdorff distance.

3.4. Lemma. For any two subsets $A, B$ of $X$ their Hausdorff distance associated with the cosmic distance $d^{c}$ satisfies

$$
d\left(R(A)_{1}, R(B)_{1}\right) \leq d^{c}(A, B) \leq 2 d\left(R(A)_{1}, R(B)_{1}\right) .
$$

If $X$ is a Hilbert space the following equality holds:

$$
d^{c}(A, B)=d\left(R(A)_{1}, R(B)_{1}\right) .
$$

Proof. Given $r>d^{c}(A, B)$, for each $a \in A$ we can find $b \in B$ with $d^{c}(a, b)<$ $r$. Thus

$$
e\left(R(a)_{1}, R(B)_{1}\right) \leq e\left(R(a)_{1}, R(b)_{1}\right) \leq d^{c}(a, b)<r
$$

and $e\left(R(A)_{1}, R(B)_{1}\right) \leq r$. Using the symmetry of the roles of $A$ and $B$ we get $d\left(R(A)_{1}, R(B)_{1}\right) \leq r$ and the first inequality follows.

Now let $r>d\left(R(A)_{1}, R(B)_{1}\right)$. For each $a \in A$ we can find $b \in B$ and $t \in[0,1]$ such that $\|\hat{a}-t \hat{b}\|<r$. Then we have

$$
e\left(R(a)_{1}, R(b)_{1}\right) \leq d\left(\hat{a}, R(b)_{1}\right) \leq\|\hat{a}-t \hat{b}\| \leq r
$$

and

$$
\begin{aligned}
e\left(R(b)_{1}, R(a)_{1}\right) & \leq e\left(\hat{b}, R(a)_{1}\right) \leq\|\hat{b}-\hat{a}\| \leq(1-t)\|\hat{b}\|+\|\hat{a}-t \hat{b}\| \\
& <r+\|\hat{a}\|-t\|\hat{b}\| \leq r+\|\hat{a}-t \hat{b}\| \leq 2 r,
\end{aligned}
$$

and finally $d^{c}(a, b) \leq 2 r$. It follows that $d^{c}(A, B) \leq 2 r$.

When $X$ is a Hilbert space, for $a, b$ as above, we can find $t \in[0,1]$ such that $\|\hat{a}-t \hat{b}\|=d(\hat{a},[0,1] \hat{b})$. Then we have either $t=0$ and $(\hat{a} \mid \hat{b}) \leq$ $0, d(\hat{a},[0,1] \hat{b})=1=d(\hat{b},[0,1] \hat{a})$ or $\hat{a}-t \hat{b} \perp \hat{b}$ and

$$
\|\hat{a}-t \hat{b}\|^{2}=1-t(a \mid b)=1-(a \mid b)^{2}=\|\hat{b}-t \hat{a}\|^{2}
$$

so that $d(\hat{b},[0,1] \hat{a}) \leq d(\hat{a},[0,1] \hat{b}), d^{c}(a, b) \leq r$, and $d^{c}(A, B) \leq r$. 
Combining this result with Lemma 3.2 we get

$$
d_{1}(R(A), R(B)) \leq d^{c}(A, B) \leq 4 d_{1}(R(A), R(B)) .
$$

Using the fact that for two convex cones $P, Q$ we have

$$
d_{r}(P, Q)=r d_{1}(P, Q)
$$

we get that a sequence $\left(A_{n}\right)$ of the set $\mathscr{C}(X)$ of nonempty convex subsets of $X$ converges to $A \in \mathscr{C}(X)$ for $d^{c}$ iff the sequence $\left(R\left(A_{n}\right)\right)$ converges to $R(A)$ for the family of quasi-distances $\left(d_{r}\right)_{r>0}$. Now Proposition 1.3 and Corollary 1.4 imply that the mapping $A \rightarrow R(A)$ is a homeomorphism of $\mathscr{C}(X)$ onto its image in $\mathscr{C}(X \times \mathbb{R})$ when $\mathscr{C}(X)$ and $\mathscr{C}(X \times \mathbb{R})$ are endowed with the topologies associated with $\left(d_{r}\right)_{r>0}$. Combining the previous observations we get the following result.

3.5. Theorem. The bounded Hausdorff topology on the set $\mathscr{C}(X)$ of nonempty convex subsets of $X$ (i.e., the topology induced by the family $\left.\left(d_{r}\right)_{r>0}\right)$ coincides with the topology associated to the Hausdorff cosmic distance.

Identifying a function with its epigraph this result can be transferred to the space of convex functions on a n.v.s. On the other hand, using Theorems 2.3 and 3.5 we get immediately the following consequence proved in [2, Theorem 4.6] for Hilbert spaces.

3.6. Corollary. For any n.v.s. $X$, the polarity is a continuous mapping from $\mathscr{E}(X)$ into $\mathscr{C}\left(X^{*}\right)$ when $\mathscr{C}(X)$ and $\mathscr{C}\left(X^{*}\right)$ are endowed with the cosmic distance.

Conversely, Theorem 3.5 shows that, in the Hilbert case, the continuity results of [13] and our Theorem 2.3, Corollary 2.4 are consequences of [2, Theorem 4.6].

\section{ACKNOWLEDGMENTS}

The author would like to thank the referee for a meticulous reading of the manuscript, in particular for detecting an error in the first version of Lemma 3.4 and for several helpful suggestions concerning the presentation of the paper.

\section{REFERENCES}

1. H. Attouch, Variational convergence for functions and operators, Applicable Maths. series, Pitman, London, 1984.

2. H. Attouch and R.J.-B. Wets, Isometries for the Legendre-Fenchel transform, Trans. Amer. Math. Soc. 296 (1986), 33-60.

3. __ Another isometry for the Legendre-Fenchel transform, J. Math. Anal. Appl. 131 (1988), 404-411.

4. __ Quantitative stability of variational systems I. The epigraphical distance, working paper, I.I.A.S.A., Laxenburg, Austria, 1988.

5. __ Quantitative stability of variational systems II. A framework for nonlinear conditioning, working paper, I.I.A.S.A., Laxenburg, Austria, 1988. 
6. D. Azé, On some metric aspects of set convergence, Univ. Perpignan, preprint, 1988.

7. D. Azé and J.-P. Penot, Operations on convergent families of sets and functions, Optimization 21 (1990).

8. Recent quantitative results about the convergence of convex sets and functions, Functional analysis and approximation (P. L. Papini, ed.) (Proc. Internat. Conf. Bagni di Lucca, 1988; Pitagora, Bologna, 1989), pp. 90-110.

9. D. Azé, J.-P. Penot, and M. Volle, Qualitative results about the convergence of convex sets and convex functions, in preparation.

10. G. Beer, On Mosco convergence of convex sets, Bull. Austral. Math. Soc. 38 (1988), 239-253.

11. _ On the Young-Fenchel transform for convex functions, Proc. Amer. Math. Soc. 104 (1988), 1115-1123.

12. Convergence of continuous linear functionals and their level sets, Arch. Math. $\mathbf{5 2}$ (1989), 482-491.

13. Conjugate convex functions an the epi-distance topology, Proc. Amer. Math. Soc. 108 (1990), 117-126.

14. G. Beer and R. Lucchetti, Continuity results for the epi-distance topology with applications to constrained convex optimization problems, preprint.

15. Ch. Castaing and M. Valadier, Convex analysis and measurable multifunctions, Lecture Notes in Math., vol. 580, Springer-Verlag, 1977.

16. L. Contesse, On the continuity of polar point-to-sets maps, Publ. A.N.O., vol. 55, Univ. Lille, 1981.

17. L. Contesse and J.-P. Penot, Continuity of the Fenchel correspondence and continuity of polarity, J. Math. Anal. Appl. (to appear).

18. A. Fougères, Coercivité des intégrandes convexes normales. Application à la minimisation des fonctionnelles intégrales et du calcul des variations (I). Travaux du Séminaire d'analyse convexe, Montpellier, 1976, exposé no. 19.

19. J.-B. Hiriart-Urruty, A general formula on the conjugate of the difference of functions, Canad. Math. Bull. 29 (1986), 482-485.

20. L. Hormander, Sur la fonction d'appui des ensembles convexes dans un espace localement convexe, Ark. Mat. 3 (1954), 181-186.

21. J.-L. Joly, Une famille de topologies sur l'ensemble des fonctions convexes pour lesquelles la polarité est bicontinue, J. Math. Pures Appl. (9) 52 (1973), 421-441.

22. P. Huard, Une approche synthétique des recherches linéaires dans les méthodes de pente, Publ. A.N.O. vol. 44, Univ. Lille, 1981.

23. D. G. Luenberger, Optimization by vector space methods, J. Wiley, New York, 1969.

24. U. Mosco, Convergence of convex sets and of solutions of variational inequalities, Adv. in Math. 3 (1969), 510-585.

25. $\ldots$, On the continuity of the Young-Fenchel transform, J. Math. Anal. Appl. 35 (1971), 518-535.

26. J.-P. Penot, On regularity conditions in mathematical programming, Math. Programming Stud. 19 (1982), 167-199.

27. _ Preservation of persistence and stability under intersections and operations, J. Optim. Theory Appl. (to appear).

28. __ Topologies and convergences on the set of convex functions, Nonlinear Anal. (to appear).

29. R. T. Rockafellar, Convex Analysis, Princeton Univ. Press, Princeton, New Jersey, 1970. 
30. M. Volle, Sur une forme de continuité du passage au cône polaire, private communication.

31. D. W. Walkup and R. J.-B. Wets, Continuity of some convex-cone-valued mappings, Proc. Amer. Math. Soc. 18 (1967), 229-235.

32. R. J.-B. Wets, Convergence of convex functions, variational inequalities and convex optimisation problems (R. Cottle, F. Gianessi, J.-L. Lions, eds.), J. Wiley, New York, 1980.

Département De Mathématiques, Faculté Des Sciences, Avenue De L'Université, 64000 PAU, FRANCE 